Revue des patrimoines

16 | 2011

Le patrimoine militaire et la question urbaine

\title{
La Manufacture d'Armes de Saint-Étienne : un patrimoine militaire saisi par l'économie créative
}

Thomas Zanetti

\section{(2) OpenEdition}

Journals

Édition électronique

URL : http://journals.openedition.org/insitu/206

DOI : 10.4000/insitu.206

ISSN : 1630-7305

Éditeur

Ministère de la Culture

Référence électronique

Thomas Zanetti, «La Manufacture d'Armes de Saint-Étienne : un patrimoine militaire saisi par l'économie créative », In Situ [En ligne], 16 | 2011, mis en ligne le 27 juillet 2011, consulté le 10 décembre 2020. URL : http://journals.openedition.org/insitu/206 ; DOI : https://doi.org/10.4000/insitu 206

Ce document a été généré automatiquement le 10 décembre 2020.

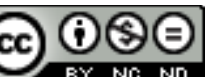

In Situ Revues des patrimoines est mis à disposition selon les termes de la licence Creative Commons Attribution - Pas d'Utilisation Commerciale - Pas de Modification 4.0 International. 


\title{
La Manufacture d'Armes de Saint- Étienne : un patrimoine militaire saisi par l'économie créative
}

\author{
Thomas Zanetti
}

\section{Introduction}

1 Saint-Étienne est une ville caractérisée par son glorieux passé industriel. Le développement urbain du chef-lieu de la Loire est avant tout issu du développement de diverses activités (textile, armurerie, passementerie, quincaillerie, sidérurgie) qui prennent leur véritable essor durant le XIXe siècle. La ville contemporaine se fonde sur la croissance industrielle et le paysage urbain porte rapidement les marques des activités dominantes. L'armurerie stéphanoise constitue une branche particulièrement dynamique depuis le regroupement, en 1764, de neuf fabricants en Manufacture Royale ayant le monopole de la fourniture des armes pour le Roi. À partir des années 1817-1820, la mise en marche de l'essor industriel commence, et, entre les années 1920 et les années 1960, l'économie stéphanoise connaît son apogée ${ }^{1}$. L'armurerie s'organise alors principalement à partir de la Manufacture, devenue propriété de l'État en 1838, et qui représente le principal établissement d'une ville progressivement élevée au rang d'arsenal ${ }^{2}$ de la France en guerre. Le titre de Manufacture d'Armes de Saint-Étienne (MAS) renvoie toujours à cette époque au rassemblement de divers petits ateliers disséminés dans la ville, mais à partir de 1864 est construit sur un terrain de 12 hectares un ensemble industriel, terminé en 1870 , dans l'optique de regrouper physiquement, et non plus simplement administrativement, l'ensemble des fabricants concernés. La manufacture a ensuite été étendue en 1887 et en 1889, avec l'adjonction de nouveaux bâtiments. Les agrandissements se sont d'abord faits en surface, puis en hauteur, jusqu'à porter la taille de l'ensemble à 19 hectares. Après une phase de croissance régulière, le secteur des armes va connaître un fort développement durant le premier conflit mondial, qui se répétera lors de la Seconde Guerre mondiale. Les effectifs successifs traduisent l'importance de cet ensemble industriel : 10000 ouvriers en 1890, 16000 durant la Première Guerre mondiale, 
10000 en 1940. Après un lent mais inévitable déclin, l'établissement, pilier symbolique de l'économie locale, fermera en 1990, dans un contexte de crise de l'industrie de l'armement. En 2000, un service d'archives militaires s'implante sur le site dans le but de sauvegarder des emplois. À partir du milieu des années 1970, la situation économique à Saint-Étienne est celle d'une crise d'une puissance sans précédent et, durant la décennie 1980, l'agglomération stéphanoise représente le type même d'une vieille région industrielle très durement touchée par la crise économique. Le paysage urbain en porte désormais les stigmates, notamment avec la multiplication des friches industrielles et des espaces urbains déshérités. Il n'est ainsi pas surprenant de constater que le patrimoine stéphanois est dominé par un héritage issu des $\mathrm{XIX}^{\mathrm{e}}$ et $\mathrm{XX}^{\mathrm{e}}$ siècles. Les éléments architecturaux et urbains, mais également tout un patrimoine social, culturel et technique, considérés dans un premier temps comme autant de stigmates rappelant le déclin de l'industrie locale, sont aujourd'hui identifiés comme des traces à conserver et à mettre en valeur.

\section{Les valeurs patrimoniales d'un haut lieu de l'industrie locale}

\section{Le registre architectural et urbain}

2 La construction de la Manufacture d'Armes de Saint-Étienne s'est déroulée sur six années, au cours de trois différentes phases, mais son plan d'ensemble a été conçu entièrement de manière géométrique. La manufacture n'a subi, hormis quelques surélévations, aucun changement depuis son édification, ce qui permet d'avoir aujourd'hui un héritage presque intact, dans un parfait état de conservation, et dont l'organisation initiale a été préservée. À la fois de facture classique et contenant un caractère original, le site a été construit tel un échiquier ou un damier intégrant espaces pleins et espaces vides. La morphologie urbaine de l'ensemble fait ainsi apparaître une véritable cité industrielle dans l'esprit de la pensée utopiste, et de l'expression de ce que pouvait être une cité industrielle idéale sous le Second Empire ${ }^{3}$. (fig. $\mathbf{n}^{\circ} \mathbf{1}$ ) 


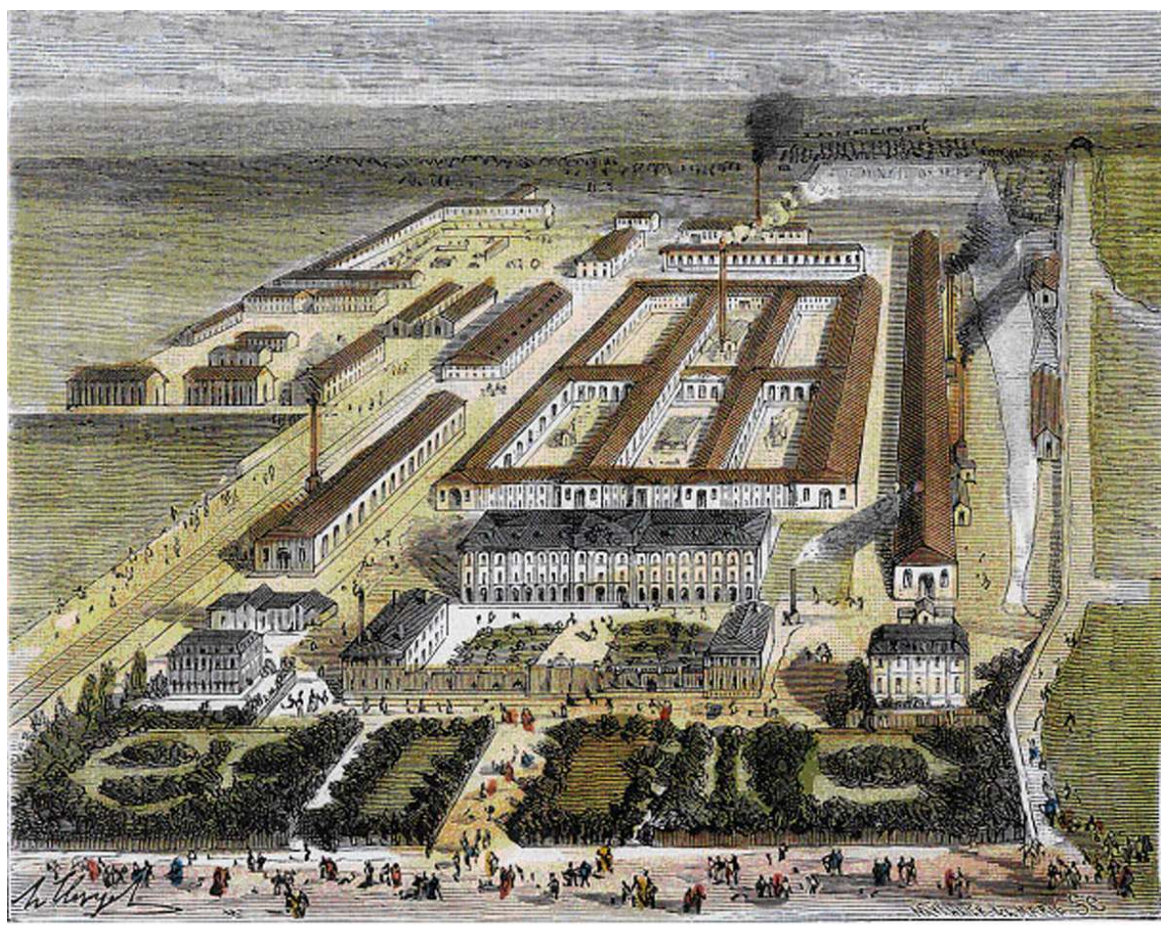

Manufacture Impériale d’armes

Saint.Etienne (1866-1868)

Dessin représentant la Manufacture au XIXe siècle.

(c) Association ARCO, juin 2005.

La réalisation du site entraîne en effet la composition d'un espace urbain et industriel uniforme et homogène, selon un ordonnancement et un tracé symétriques, et la MAS tire de cette cohérence générale son aspect le plus original. Les bâtiments édifiés pour la manufacture possèdent un style architectural reconnaissable, typique du Second Empire, grâce à l'utilisation privilégiée des briques et de la pierre blanche. L'emploi prédominant de ces matériaux pour les deux hectares de construction présents ajoutent à l'uniformité et au caractère monumental du lieu. La manufacture apparaît donc comme un élément remarquable de l'architecture industrielle du XIX siècle, et elle est considérée à ce titre comme un ensemble architectural unique pour la ville de Saint-Étienne. Il existe également au sein de la MAS une distribution fonctionnelle des espaces autour de la cour d'honneur centrale, selon les rôles qui leur sont attribués. Les lieux de production sont constitués par les ateliers regroupés dans trois longs corps de bâtiments encadrant une construction en « double $\mathrm{H}$ ", qui abrite en son centre le pavillon équipé d'une machine à vapeur. La grande fabrique du « double $\mathrm{H}$ », dont les dimensions conséquentes, $115 \mathrm{~m} \mathrm{x}$ $118 \mathrm{~m}$, lui confèrent un aspect monumental, renferme pour sa part cinq cours pavées. Éléments essentiels de l'activité du site, les bâtiments productifs sont disposés en son centre, de part et d'autre de la cour d'honneur. Les lieux de vie et d'habitation des travailleurs (cantine, dortoirs, réfectoire) sont quant à eux regroupés dans les parties plus périphériques de l'ensemble industriel, à ses extrémités Nord et Ouest. Les édifices réservés à l'administration de la MAS sont enfin agencés à l'entrée du site, de part et d'autre de la cour d'honneur. Ils se composent des maisons abritant la direction, soit un hôtel particulier pour le directeur et un second pour le sous-directeur, qui encadrent avec leurs jardins suspendus une esplanade légèrement inclinée de $100 \mathrm{~m}$ x $70 \mathrm{~m}$ où l'on trouve 
également deux autres bâtiments administratifs. La transition vers les espaces dédiés à la production est signalée par le bâtiment dit "de l'Horloge ", qui symbolise le passage des domaines administratifs aux parties industrielles du site. Il existe donc, à travers la morphologie urbaine de la MAS, une hiérarchie socio-spatiale qui renforce la cohérence générale ayant présidé à sa conception. L'ensemble est délimité par une grille de $78 \mathrm{~m}$ de long, rehaussée d'un majestueux portail surmonté des aigles impériaux marquant l'entrée de l'usine. Pour toutes ces qualités que nous avons évoquées, la manufacture est ainsi appréhendée, aussi bien localement qu'au niveau national, comme un chef-d'œuvre de l'architecture militaire.

\section{Le registre technique}

4 À l'époque de la réalisation de la MAS, on projette l'adoption des principes d'organisation rationalisée du travail, avec notamment une conception scientifique et un contrôle de la production. Les lieux consacrés à la fabrication des armes auraient ainsi été le cadre de la mise en place progressive d'une division des tâches poussée qui aboutira ensuite au taylorisme. Ils sont donc liés à un projet industriel de grande envergure qui se définit autour de l'ambition naissante d'une production de masse. Les espaces productifs occupent également une place importante dans l'histoire du site car les armes issues des ateliers ont acquis au fil du temps une réputation utilitaire et esthétique ${ }^{4}$ considérable. L'activité industrielle est ainsi caractérisée par une tentative de concilier production en série et intégration d'un certain degré de création artistique. Cette recherche de dialogue entre l'art et l'industrie renvoie à la définition de l'activité du design, dont les fondements semblent être au cœur du rôle de l'École des beaux-arts de Saint-Étienne, créée en 1857. Cette dernière a alors pour mission d'améliorer les produits industriels et de promouvoir l'innovation, de susciter une intégration de l'esthétique dans la production, de faciliter la rencontre entre les savoir-faire des ouvriers et la création artistique. Cette tradition de technicité, de qualification, d'esprit de créativité et d'inventivité est également inscrite dans l'histoire de la MAS. En 1866, avec la construction de la nouvelle manufacture, l'industrie de l'armement engage une recherche de l'esthétique et des formes nouvelles tout en procédant à une organisation plus intense de la production: contrôle du travail, apparition de bureaux d'études, de laboratoires d'essais, début du développement de la standardisation. Ce lien entre rationalisation industrielle et considération plus importante pour l'aspect visuel est alors appréhendé comme participant de l'ébauche de la notion contemporaine du design. Cela aurait d'ailleurs été une constante de l'histoire de la MAS, puisque près de 100 ans plus tard, le FAMAS (fusil d'assaut de la MAS), qui a fait la fierté des ouvriers du site, en plus d'être à la pointe du progrès, possède une forme originale et un design industriel novateur. Les bâtiments de production de l'ensemble sont donc porteurs d'un sens qui renvoie à l'histoire économique et aux techniques introduites lors de la révolution industrielle, du recours aux machines-outils jusqu'à la conception scientifique mobilisée pour la croissance de la production. La situation de la manufacture à l'échelle de la commune est elle aussi révélatrice, par la proximité géographique qu'elle entretient avec la première ligne de chemin de fer française, des bouleversements instaurés par les progrès des transports, intimement liés aux évolutions économiques. 


\section{Le registre social et culturel}

5 La manufacture occupe une place particulière chez les Stéphanois, tout d'abord par la taille de l'établissement et par le niveau régulièrement atteint par ses effectifs au cours de son histoire. Les habitants de la ville ont ainsi généralement eu dans leur famille ou dans leur cercle de connaissance une personne travaillant sur le site, ce qui a entraîné une certaine familiarité. Le statut d'enceinte militaire protégée par le secret défense a ensuite pu conférer une part de mystère au site et à l'activité qui s'y est déroulée. Élément essentiel du riche passé industriel stéphanois, elle évoque le rôle primordial joué par la classe ouvrière dans l'histoire sociale locale. Si la classe ouvrière de Saint-Étienne se caractérise par sa diversité issue de la grande variété des groupes et des univers professionnels ${ }^{5}$, une tradition de solidarité s'institue parmi les populations laborieuses à partir du milieu du XIX ${ }^{e}$ siècle. Ainsi, des avancées sociales apparaissent avec l'avènement du mouvement mutualiste à partir de 1855 , et la ville est alors un des berceaux de naissance des secours mutuels qui tentent d'apporter des solutions à la dureté des conditions de vie des classes les plus défavorisées. Cette histoire industrielle porte ensuite l'empreinte des combats menés par les ouvriers dans le cadre du développement du syndicalisme. Au cours des années 1917-1920, plusieurs grèves éclatent successivement. La contestation s'inscrit dans un contexte de dégradation du cadre d'existence des travailleurs, avec notamment la multiplication des taudis et des logements insalubres, et d'antagonisme social autour de la question des salaires versés par les patrons. Il règne alors à Saint-Étienne une agitation sociale qui conduit le mouvement ouvrier à s'intensifier et on assiste à une multiplication des épisodes conflictuels. Cependant, on peut avancer que la mémoire sociale de cet espace industriel reste très spécifique, voire contrastée. En effet, les employés de la manufacture étaient généralement des ouvriers d'État, recrutés sur concours, plutôt bien rémunérés et formant ainsi une élite, une sorte d'aristocratie ouvrière. Dès 1915, certains ouvriers spécialistes de la MAS gagnaient jusqu'à 30 francs par jour alors qu'en moyenne le salaire d'un ajusteur était de 6 francs. Il existait donc une relative distance entre les divers groupes professionnels, ce qui a même pu créer une certaine jalousie envers les plus favorisés d'entre eux; toutefois le passé ouvrier de la cité stéphanoise reste dominé par les pratiques d'entraide et de camaraderie. Sur le site de la MAS, il existe des traces de cette histoire sociale, telles que le monument aux morts (fig. $\mathbf{n}^{\circ} \mathbf{2}$ ) situé devant le portail d'entrée de la MAS. Entièrement financé par des travailleurs, il est représentatif de la population ouvrière locale et de ses contradictions qui mêlent unicité et diversité. Les maisons de la direction, en inscrivant spatialement la présence de l'autorité, constituent quant à elles le symbole de la hiérarchie sociale à l'œuvre sur le site. Celui-ci est enfin porteur d'une mémoire plus sombre, celle des guerres et des conflits auxquels a participé l'industrie d'armement, cette dernière renvoyant alors à l'aspect lourd des événements associés. 


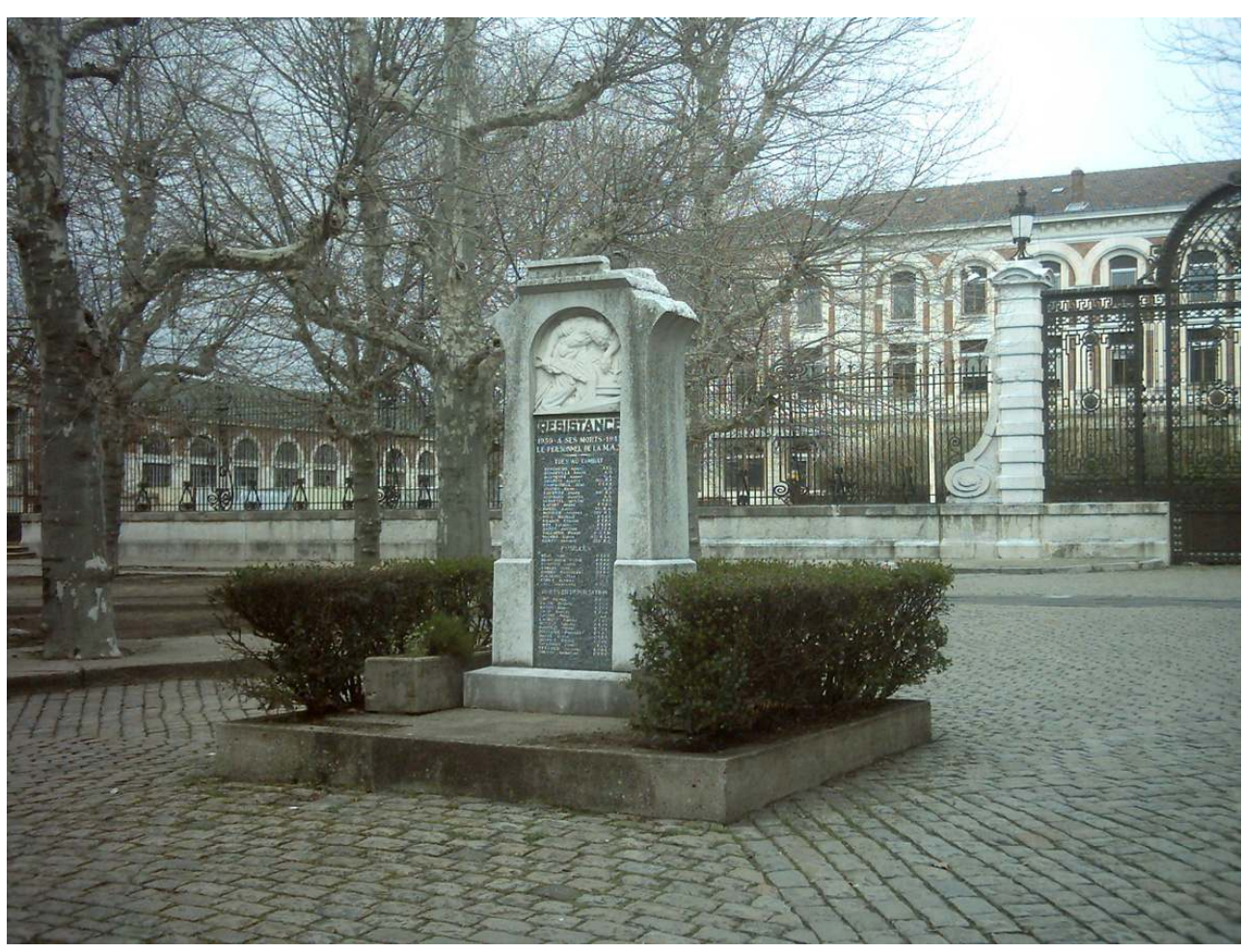

Le monument aux morts situé à l'entrée de la Manufacture.

Phot. T. Zanetti, 2006.

\section{Héritage industriel et enjeux urbains contemporains}

\section{Une agglomération « en perte de vitesse »}

Depuis la période de crise qui a vu l'effondrement des piliers industriels de l'agglomération, celle-ci a connu un déclin démographique, économique, social et urbain. Le recensement de 1982 fait ainsi apparaitre une diminution de la population de près de 20000 habitants par rapport à celui de 1968, les pertes se poursuivant jusqu'à nos jours. On observe également un fort accroissement du nombre de logements vacants et une montée préoccupante du taux de chômage. La situation économique à Saint-Étienne est donc celle d'une crise d'une ampleur sans précédent, et la région vit une récession qui frappe les principaux secteurs industriels et conduit à l'effondrement des bases traditionnelles de l'activité locale. Au cours des années 1980, l'agglomération stéphanoise représente le type même d'une « shrinking city ${ }^{6}$ » ou d'une vieille région industrielle très durement touchée par la crise économique. L'économie locale, dont la base est composée d'un grand nombre d'entreprises proches de l'artisanat, devait sa montée en puissance à quelques importants groupes dont la création avait souvent été directement stimulée par l'État dans la logique des champions nationaux ${ }^{7}$. Le tissu industriel s'est retrouvé profondément déstabilisé par le départ des donneurs d'ordres qui assuraient, au sein d'un système de relations verticalement intégré, une activité régulière à une multitude de PME et de PMI dépendantes financièrement et technologiquement. Le paysage urbain a rapidement porté les stigmates de la dégradation économique, avec la multiplication de 
friches industrielles et d'espaces en déshérence. Ces derniers ont dans un premier temps fait l'objet d'une politique de tabula rasa et n'étaient pas encore appréhendés comme autant d'opportunités de recréer la ville sur elle-même. La priorité fut tout d'abord donnée à la sauvegarde de l'emploi, même si l'on note la mise en œuvre, dès le début des années 1990, d'initiatives en faveur de la réhabilitation et de la muséification d'anciennes emprises industrielles ${ }^{8}$. L'agglomération est alors caractérisée par une relative instabilité politique $^{9}$ et la ville voit se succéder à sa tête quatre maires en trente années. De cette inconstance chronique naissent des conditions économiques, sociales et politiques ne permettant pas l'émergence d'un acteur collectif disposant d'une légitimité suffisamment importante pour imposer un vaste projet de reconversion à l'échelle du territoire. Aujourd'hui encore, l'absence à Saint-Étienne d'un régime de gouvernement urbain durable empêche l'inscription à l'agenda politique local d'un projet global et consensuel de reconversion de l'économie industrielle disparue. Toutefois, après l'élaboration d'une politique strictement économique et d'opérations d'aménagement du cadre urbain, on observe depuis la fin des années 1990 le déploiement d'un projet plus général d'attractivité territoriale. Aujourd'hui, le renouvellement urbain a donc remplacé de manière définitive les politiques de planification et d'incitation à l'implantation d'entreprises.

\section{Le défi de l'attractivité métropolitaine}

Dans un contexte de concurrence métropolitaine exacerbée, de « régions qui gagnent $»^{10}$ et de territoires qui restent à la périphérie de la croissance, la ville de Saint-Étienne s'est lancée dans un processus de production d'une image positive et valorisante dans le but d'attirer les investisseurs, elle qui souffre toujours de son image de « ville noire ». Les élus ont ainsi entrepris d'insuffler à l'agglomération une nouvelle identité empreinte de modernité. L'arrivée à la tête de l'instance municipale d'un maire entrepreneur ${ }^{11}$, qui va personnifier le nouveau positionnement économique et urbain stéphanois, coïncide avec l'identification de leviers inédits afin de redonner à l'agglomération un dynamisme qui lui fait défaut. Ces instruments devant permettre à l'agglomération de se relancer dans la course à la réussite métropolitaine s'articulent autour des notions de compétitivité, d'attractivité, de consommation, d'offre territoriale, de culture et d'identité locale, et mettent en œuvre des mécanismes de marketing urbain. Elles traduisent la volonté de procéder à une adaptation des bonnes pratiques urbaines et architecturales en circulation dans les anciennes villes industrielles des pays développés. La contextualisation des normes internationales doit alors donner lieu à la conception d'un modèle local spécifique, entre l'emprunt et le dépassement ${ }^{12}$ des cas de réussite ayant valeur de référence. Les élus stéphanois ne cachent alors pas leur intention de s'appuyer sur le célèbre exemple de Bilbao qui, en accueillant le musée Guggenheim, a pu insuffler une revalorisation foncière et symbolique à un territoire déqualifié et stigmatisé. Le recours à la thématique culturelle semble alors faire partie de la "recette " pour impulser une dynamique de développement économique et en garantir la bonne visibilité. La production d'une image globale localisée s'articule ensuite autour du référentiel de qualité urbaine qui domine l'action publique contemporaine. L'opportunité foncière et symbolique induite par l'existence à Saint-Étienne de nombreuses friches industrielles peut dorénavant être saisie pour entraîner la mutation globale de tout un quartier. L'avènement d'une identité territoriale originale est ainsi amené à se matérialiser dans un projet de renouvellement architectural, urbain et paysager qui aspire à attirer les 
classes moyennes et supérieures, ou encore " créatives $»^{13}$, nécessaires pour provoquer un mouvement de mixité sociale "par le haut ». Il existe en effet un lien fréquent entre la réhabilitation d'anciens espaces industriels et la «gentrification» des quartiers concernés.

\section{La Cité du Design : symbole du renouveau stéphanois}

8 Au sein de ce tournant pris par l'action publique stéphanoise, le projet de la Cité du Design, qui se déploie sur le site de la Manufacture d'Armes de Saint-Étienne, témoin du glorieux passé industriel de la ville, tient une place centrale. Ce projet urbain, considéré comme un «moteur de dynamisme pour le redéploiement économique vers le secteur tertiaire et l'international, et le moteur d'une nouvelle urbanité et d'une nouvelle image, tournées vers la modernitét ${ }^{14} »$, s'appuie donc sur un équipement collectif de grande envergure. L'enveloppe physique de la Cité, qui consiste en l'édification d'une platine aux dimensions imposantes, doit signifier dans le paysage, au moyen d'une architecture iconique, le retournement identitaire espéré. Le lauréat du concours a ainsi proposé pour le bâtiment de la future Cité du Design un véritable signe architectural, dont la modernité s'exprime à travers les matériaux utilisés. La définition conceptuelle large de la Cité du Design traduit une vocation à rassembler tous les acteurs de ce secteur au sein d'un programme fédérateur sur la culture de l'objet et le développement de la création industrielle, par la promotion d'un triptyque "formation/recherche/création». Ambitionnant de devenir un acteur majeur de la mutation économique de la région, le nouvel équipement compte porter auprès des entreprises l'image d'un design stratégique, au service de la croissance et de la performance, ceci afin de les aider dans l'élaboration de stratégies industrielles créatives fondées sur la formation des jeunes designers et sur l'invention d'outils innovants. Inaugurée en octobre 2009, la Cité du Design mêle donc des objectifs en direction des milieux professionnels et des aspirations touristiques à destination du grand public. Elle se veut à ce sujet le vecteur de diffusion le plus efficace et visible d'une " culture design " dans l'ensemble de la société locale, et entretient pour cela des partenariats de recherche avec le milieu universitaire stéphanois, particulièrement avec l'École supérieure d'art et de design de Saint-Étienne. En choisissant la MAS comme lieu d'implantation, la Cité du Design a également voulu instaurer un dialogue entre la modernité architecturale du projet retenu et l'héritage patrimonial transmis par les bâtiments industriels. Placée à l'entrée du site, entre le portail et le bâtiment dit « de l'Horloge »" ${ }^{15}$, la Cité prétend être le témoignage d'une culture collective, du changement d'image de la ville, de la transition vers une nouvelle phase de croissance économique, et de la continuité historique de l'activité du design sur le même lieu. (fig. $\mathbf{n}^{\circ} \mathbf{3}$ ) 


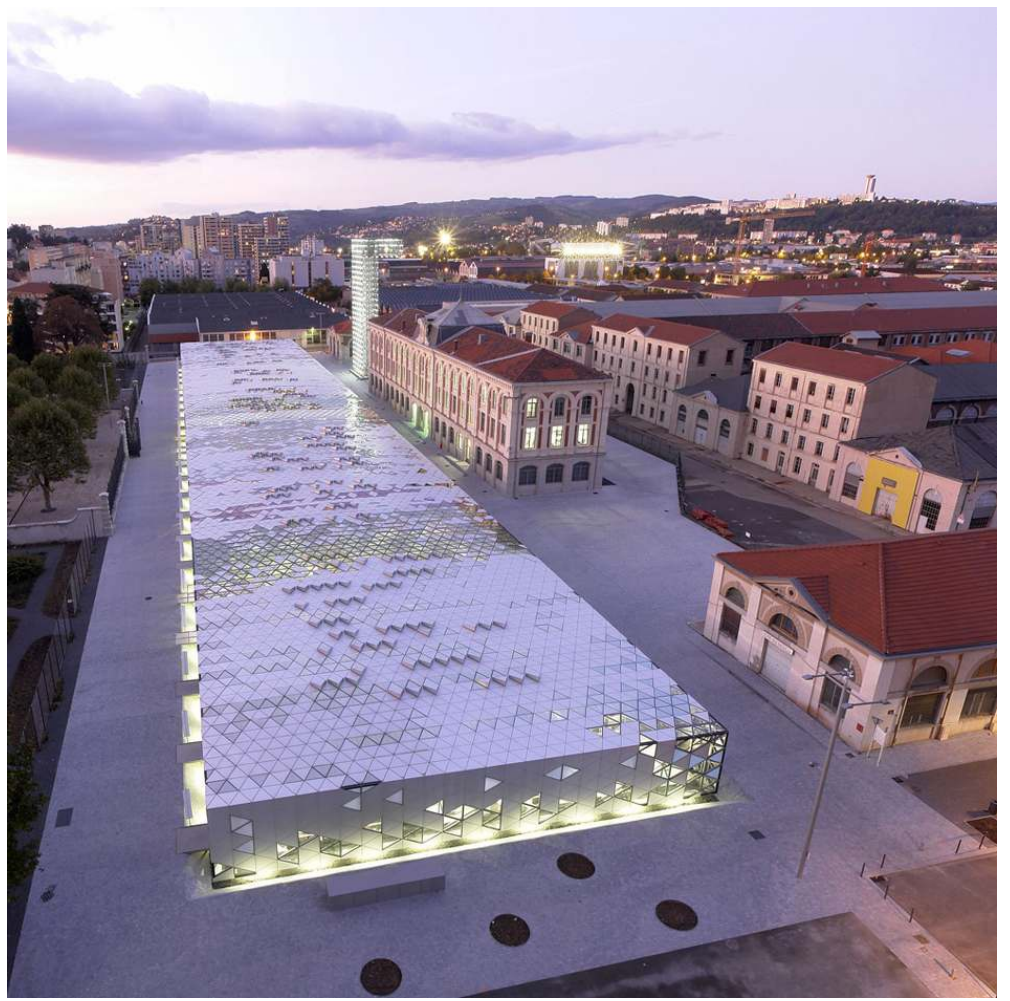

La Cité du Design dans son environnement patrimonial et urbain.

(c) The Architectural Review, 5 novembre 2009.

\section{Une patrimonialisation conflictuelle}

\section{La consécration de l'héritage technique}

La patrimonialisation de la Manufacture d'Armes de Saint-Étienne, en tant que mécanisme social et politique de production mémorielle, a donné naissance à un conflit patrimonial et mémoriel qui a traversé l'ensemble de la société locale. En effet, en vue de libérer l'espace devant accueillir la future Cité du Design, les bâtiments administratifs et les maisons de la direction (fig. $\mathbf{n}^{\circ} \mathbf{4}$ ) placés à l'entrée du site ont été démolis. La polémique a pris sa source lors de la présentation du projet architectural retenu, lorsque plusieurs associations stéphanoises de défense et de promotion du patrimoine se sont vivement émues en constatant que l'édification de la Cité du Design nécessitait la destruction de ces quatre bâtiments. Une procédure controversée de patrimonialisation de la Manufacture a ensuite conduit à l'inscription partielle de celle-ci au titre de l'Inventaire supplémentaire des Monuments historiques. Le classement concerne ainsi la totalité de l'ensemble industriel, à l'exception des anciens locaux de l'administration du site. Ce processus d'élection patrimoniale, qui évalue l'héritage à transmettre et les traces à conserver, traduit une volonté de favoriser la mémoire technique contenue dans les installations productives de la manufacture. Les élus et responsables de la Cité ont donc procédé à une entreprise de mobilisation du passé selon les enjeux du présent, attribuant au lieu une série de valeurs à même de justifier les choix engageant l'avenir du territoire, et de les inscrire dans une continuité historique sécurisante. En réinvestissant le passé de 
la Manufacture à l'aune d'une mémoire technique nourricière, les institutions politiques locales ont pu élever Saint-Étienne au rang de berceau de l'activité du design. Le potentiel signifiant de la Manufacture est utilisé au service du projet Design car il véhicule certaines valeurs positives: technologie, innovation, esthétisme, art. L'exercice de remémoration des savoir-faire déployés lors du processus de fabrication des armes de la MAS devient une réserve de sens ${ }^{16}$, et le recours au patrimoine industriel peut alors être appréhendé comme un mécanisme de légitimation de l'action publique contemporaine, avec le risque qu'il conduise ainsi à une manipulation de l'esprit du lieu hérité. Dans le but de spécifier localement les «bonnes pratiques» urbaines en circulation, les collectivités sont amenées à s'appuyer sur leur histoire, sans toutefois s'exposer à un éloignement des normes internationales en vigueur qui garantissent sa visibilité au projet de renouvellement urbain. Ce caractère collusif entre mise en valeur patrimoniale et mise en œuvre d'un référentiel «design ", en tant que levier d'action des objectifs portés à l'agenda local, se manifeste dans la communication municipale par un glissement sémantique éloquent qui concerne le label «Ville d'Art et d'Histoire », devenu dans certains documents officiels «Ville de Design et d'Histoire». Le processus de patrimonialisation fait donc apparaître une intention de relecture et de réécriture de l'histoire locale à l'aune des enjeux induits par un contexte de recherche d'attractivité urbaine et territoriale.

Figure 4

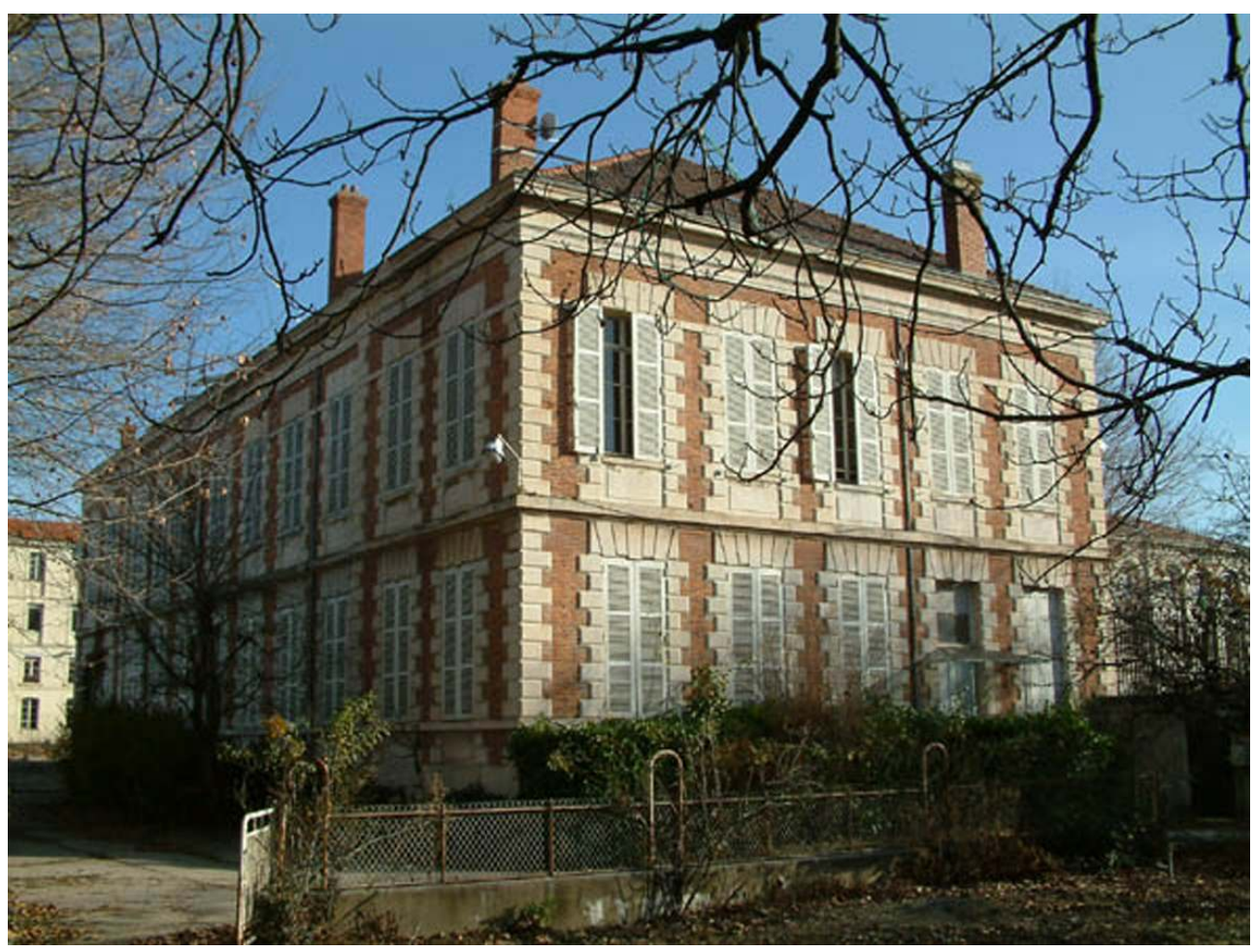

Un des bâtiments administratifs du site industriel.

Phot. T. Zanetti, 2006. 


\section{L'éviction de la mémoire ouvrière}

10 Toutefois, l'exercice de remémoration inhérent à l'action patrimoniale apparaît sélectif puisqu'il occulte de diverses manières certains pans d'une histoire douloureuse ${ }^{17}$, celle de la perte de l'activité industrielle qui a construit le territoire et fait sa richesse pendant plusieurs siècles, avant de l'entraîner vers le chômage et le déclin démographique. Un exemple de cette éclipse mémorielle nous est fourni par la publication ${ }^{18}$ parue dans le but d'officialiser l'histoire de la MAS, et qui fait la part belle à la dimension technologique de son passé. La destruction des maisons de direction, en faisant disparaître les symboles de la structure socio-spatiale inscrite sur le site et du pouvoir hiérarchique présent sur le lieu, scelle l'oubli de ce qui divise ${ }^{19}$, soit les événements conflictuels classiquement associés au monde du travail (grèves, manifestations ouvrières...). Cette histoire ouvrière stéphanoise qui reste embarrassante pour les élus locaux, car peu conforme à la nouvelle identité dynamique et moderne dont ils font la promotion, et l'existence d'une certaine nostalgie liée à l'ancienne activité industrielle, ont été désavouées dans le cadre de la réhabilitation privilégiée d'un héritage technique considéré comme une plus-value mémorielle. L'évacuation des connotations sociales du site dans le cadre de la patrimonialisation a alors été vécue par la classe ouvrière locale comme un puissant sentiment de dépossession et de mise à distance. L'éviction de sens issue de la procédure partielle d'inscription de la MAS montre ensuite la supériorité des élites patrimonialisantes sur le groupe social ouvrier local, qui souffre d'une crise de visibilitéz et peine à se réinventer une conscience de classe. La transmission de l'esprit du lieu à travers son réinvestissement par la puissance aménageuse s'est donc débarrassée de la fraction douloureuse de son potentiel signifiant, encore mal assumée par les édiles stéphanois. On assiste ainsi à l'émergence d'épisodes amnésiques et de véritables trous de mémoire qui affleurent lors de la narration de l'histoire du lieu, officialisée par la mise en patrimoine. Une construction mémorielle sélective a été produite dans le but de dessiner les contours d'une histoire locale du design, ou plus vraisemblablement d'une histoire locale au service du design. Cette dernière, dont on ne peut que souligner la visée hégémonique, a été établie par le groupe porteur de mémoire le plus éminent, à savoir l'instance politique de décision qui a démontré ainsi son contrôle de la redéfinition du passé dans le présent et sa faculté à se poser en seul juge de la «rentabilité du temps qui passe $^{21} »$.

\section{La difficile émergence d'une gouvernance patrimoniale locale}

11 Afin d'éviter le démembrement de la Manufacture, plusieurs associations se sont lancées dans une opposition au projet urbain, qui a donné naissance à un véritable conflit mémoriel au sein de la nébuleuse patrimoniale locale, voire parmi l'ensemble de la société stéphanoise. Malgré une année de combat, de demandes répétées de justice mémorielle émanant de la sphère associative, il fut décidé d'implanter la Cité du Design sur l'esplanade de la Manufacture et de procéder à la démolition des édifices concernés.

12 L'existence d'un conflit entre les habitants de la ville représentés par les associations de défense du patrimoine et les élus à l'initiative du projet, accompagnés des responsables techniques, illustre les rapports de pouvoir qui s'expriment en filigrane du projet de renouvellement urbain et de patrimonialisation. La controverse patrimoniale autour de la Manufacture et de l'implantation de la Cité du Design doit donc faire l'objet d'une analyse 
en termes d'appropriation symbolique de l'espace hérité. Les associations, véritables entrepreneurs de mémoire locale ${ }^{22}$, ont ainsi produit un discours expert justifié par un travail de connaissance historique du site en faisant appel à l'érudition de divers universitaires stéphanois, puis utilisé des recours démocratiques afin d'infléchir la décision (pétitions, lettres aux différentes autorités compétentes, campagne de sensibilisation dans la presse nationale) et apporté des solutions techniques (constitution d'un argumentaire, proposition d'une localisation alternative pour la Cité). Elles ont également profité du rôle de vigile endossé par le conseil de développement de l'agglomération qui s'est saisi du projet pour rendre un avis consultatif dans le cadre de ses attributions. Mais l'équipe politique en charge de la Cité du Design n'a pas pris en compte les revendications émanant de la société civile, et l'on peut voir la défaite électorale du maire sortant et premier défenseur du projet, aux élections municipales de 2008, comme une sanction démocratique. Le cas de la manufacture signale ainsi la présence à Saint-Étienne d'une gouvernance patrimoniale locale en formation, et dont la capacité à se constituer en acteur collectif susceptible de produire un consensus portant sur l'inscription des lieux hérités de l'industrie dans une stratégie de développement métropolitain semble inaboutie. Cette lacune renvoie à la situation de fragmentation au sein du champ local d'action publique, à l'absence d'un régime urbain présidant au devenir concerté des espaces issus du passé et réinvestis dans l'avenir.

\section{Conclusion}

Alors que le site n'hébergeait plus que quelques dizaines d'emplois, la Manufacture d'Armes de Saint-Étienne est devenue un espace à fort enjeu et à forte cohabitation mémorielle où le passé a été revisité par différents acteurs de la scène patrimoniale locale. La mise en tension de mémoires portées par les associations et d'autres produites par la municipalité a conduit à un processus de mise en patrimoine qui, en tant que mécanisme social de construction de la mémoire, a eu pour conséquence l'officialisation d'une narration historique répondant à des enjeux présents. La polémique suscitée par l'implantation de la Cité du Design signale également que l'action politique en matière culturelle et patrimoniale est ici allée à l'encontre d'une vertu pourtant habituellement induite par le développement culturel, à savoir le maintien et le renforcement de la cohésion sociale d'un territoire. À Saint-Étienne, la stratégie remémorative d'évitement a en effet mené à un remaniement des souvenirs de la population locale, de manière à les accorder avec les conditions variables de son équilibre ${ }^{23}$. L'échec du dialogue entre esprit du lieu existant et création architecturale contemporaine est alors aussi celui de la construction d'une image globale de la localité. La volonté de spécifier une opération de renouvellement urbain en ayant recours aux traces matérielles et idéelles présentes sur le site n'a donc pu éviter le déclenchement d'une controverse culturelle qui aura traversé l'ensemble de la société stéphanoise. La sélection mémorielle et patrimoniale réalisée par la sphère politique traduit un double besoin contradictoire de gommer et d'invoquer son histoire. En effaçant les symboles spatiaux de la hiérarchie sociale à l'œuvre dans le monde industriel, le projet de la Cité du Design a rejeté en partie la mémoire ouvrière transmise par le site de la manufacture, et institué inévitablement des mémoires orphelines de patrimoine. 


\section{NOTES}

1. - La ville de Saint-Étienne atteint son maximum démographique en 1968, avec 223223 habitants.

2. - Saint-Étienne ayant même, pendant quelques années, été rebaptisée « Armeville » par décret.

3. - Voir le site de l'association ARCO : http://arco-image.net/avis/manu/manu_cadre.htm.

4. - Les armes gravées par les employés de la Manufacture sont parfois considérées comme de véritables chefs-d'œuvre.

5. - Certains secteurs professionnels appartiennent au monde intégré de la grande industrie (mine, sidérurgie, aciérie), tandis que d'autres relèvent de l'univers plus traditionnel de la petite entreprise héritée de l'artisanat (passementerie, cycle, rubanerie).

6. - SHORT, John, KIM, Yeong Hyun. Globalization and the city. New York : Longman, 1999.

7. - LE GALES, Patrick. La restructuration des PMI à Saint-Étienne après la crise : traces du passé et limites de l'intégration horizontale. Sociologie du travail, $n^{\circ} 48$ (1), Janvier-Mars, 2006, p. 17-36.

8. - En 1991, la création du musée de la mine au puits Couriot se donne comme mission de préserver l'identité minière du bassin de la Loire.

9. - DORMOIS, Rémi. Les coalitions dans l'analyse des politiques urbaines postkeynésiennes. Métropoles, $\mathrm{n}^{\circ} 4,2009$.

10. - BENKO, Georges, LIPIETZ, Alain. Les régions qui gagnent. Paris : PUF, 1992.

11. - HARDING, Alan. Urban Regimes land Growth Machines. Towards a Cross-National Research Agenda. Urban Affairs Quarterly, vol. 29, n 3, 1994, p. 356-382.

12. - ARAB, Nadia. À quoi sert l'expérience des autres? Bonnes pratiques et innovation dans l'aménagement urbain. Espaces et Sociétés, $n^{\circ} 131,2007$, p. 33-47.

13. - FLORIDA, Richard. The Rise of the Creative Class: And How It's Transforming Work, Leisure, Community and Everyday Life. New-York : Basic Books, 2003.

14. - DEMAZIERE, Christophe, DORSO, Franck. Deux expériences urbaines aux marges de l'Europe. Annales de la Recherche Urbaine, $\mathrm{n}^{\circ}$ 93, 2003, p. 15-21.

15. - Voir le site de The Architectural Review: http://www.arplus.com/9096/cite-du-designsaint-etienne-france-by-lin/.

16. - COLSON, Daniel, ROUX, Jacques. Héritier d'une tradition industrielle : un enjeu temporel pour la cité. Le cas du Marais à Saint-Étienne (Loire). In ENECQ, Jean-Marie (dir.). Les villes européennes de tradition industrielle. Mutations économiques et politiques urbaines. Lille : Presses universitaires de Lille, 1994, p. 205-216.

17. - RICOEUR, Paul. La mémoire, l'histoire, l'oubli. Paris : Éd. du Seuil, 2000.

18. - Éditée par le Musée d'Art et d'Industrie (MAI) de Saint-Étienne, dont la propre histoire a également été réinvestie pour le projet de la Cité du Design, notamment à travers la figure de son créateur Marius Vachon, ardent promoteur de la rencontre entre art et industrie.

19. - TISSERON, Serge. L'inconscient du monument ou une perspective psychanalytique sur le mémorial. In DEBRAY, Régis (dir.). L'abus monumental ? Paris : Fayard, 1999, p. 81-88.

20. - VERRET, Michel. La culture ouvrière. Paris : Éd. L'Harmattan, 1996.

21. - CARBALLO, Cristina, EMELIANNOF, Cyria. La liquidation du patrimoine ou la rentabilité du temps qui passe. Annales de la recherche urbaine, 2002, n' 92, p. 49-57.

22. - SAEZ, Guy. Patrimoine et associations. La Lettre ESO, $n^{\circ} 23,2005$, p. 59-62.

23. - HALBWACHS, Maurice. Les cadres sociaux de la mémoire. Paris : Éd. Albin Michel, 1925. 


\section{RÉSUMÉS}

Cet article prend pour objet d'analyse un projet de renouvellement urbain d'un espace hérité de l'activité industrielle et militaire: la Manufacture d'Armes de Saint-Étienne. L'histoire et les caractéristiques patrimoniales de ce site, à la fois dans leurs dimensions matérielles et immatérielles, sont mobilisées dans un projet urbain hautement stratégique, et donc soumis à de multiples enjeux : la Cité du Design de Saint-Étienne. Cet équipement s'insère quant à lui dans un cadre plus large de concurrence métropolitaine et de course à l'attractivité des territoires. En effet, dans une ville marquée par le traumatisme issu du déclin de l'industrie, l'existence d'un patrimoine militaire est conçue comme une opportunité de spécifier localement la mise en œuvre des "bonnes pratiques" urbaines en circulation dans les villes européennes. Les responsables de la Cité ont donc choisi un haut lieu de l'histoire industrielle et militaire locale pour accueillir le futur symbole de la modernité stéphanoise, dans le but de tirer de cette inscription patrimoniale une plus-value historique nécessaire à la réussite du projet dans un contexte de marketing urbain. Toutefois, le processus de patrimonialisation de la Manufacture d'Armes a donné naissance à un profond conflit opposant des associations locales de protection du patrimoine aux responsables de l'urbanisme. Il importe alors de questionner l'entreprise de valorisation du patrimoine militaire en analysant quels sont les héritages mémoriels associés à l'action publique urbaine contemporaine, voire instrumentés par sa stratégie de développement territorial.

This article is an analysis of a project of urban renewal concerning a space inherited from industrial and military activity: the arms factory of Saint-Étienne. The history and the heritage characteristics of the site, in both its tangible and intangible dimensions, have been mobilized in a highly strategic urban project, the City of the Design, at the meeting point of many issues. This new design centre may be understood in a wider framework of metropolitan competition and the competition for the attractiveness of territories. Indeed, in a city marked by the traumatism stemming from the decline of the industry, the existence of a military heritage is seen as an opportunity to specify locally the implementation of the " good urban practices " in circulation in European cities. The persons in charge of the City thus chose a remarkable place of the local industrial and military history to welcome the future symbol of the local modernity, with the aim of benefitting from this patrimonial inscription a historic capital gain necessary for the success of the project in a context of urban marketing. However, the process of patrimonialisation of the arms factory gave birth to a profound conflict setting local associations of protection of the heritage against the town planners. It is important to question what is involved in this enterprise of promoting military heritage: what memory and what legacy is being accentuated by contemporary urban public action, and how might this be an instrument in inter city competition. 
INDEX

Mots-clés : mémoire ouvrière, design industriel, marketing urbain, projet architectural, gouvernance patrimoniale

Keywords : workers' memories, industrial design, urban marketing, architectural project, heritage management

\section{AUTEUR}

\section{THOMAS ZANETTI}

Doctorant en géographie et urbanisme (Université Lyon 2). tomazanetti@hotmail.com 